Purdue University Purdue e-Pubs

2013

\title{
Optimization Under Uncertainty Applied to Heat Sink Design
}

\author{
K. K. Bodla \\ Purdue University \\ J. Y. Murthy \\ S V. Garimella \\ Purdue University, sureshg@purdue.edu
}

Follow this and additional works at: http://docs.lib.purdue.edu/coolingpubs

Bodla, K. K.; Murthy, J. Y.; and Garimella, S V., "Optimization Under Uncertainty Applied to Heat Sink Design" (2013). CTRC Research Publications. Paper 213.

http://dx.doi.org/DOI: 10.1115/1.4007669

This document has been made available through Purdue e-Pubs, a service of the Purdue University Libraries. Please contact epubs@purdue.edu for additional information. 


\section{Optimization Under Uncertainty Applied to Heat Sink Design}

\section{Karthik K. Bodla} Jayathi Y. Murthy

\section{Suresh V. Garimella ${ }^{1}$}

e-mail: sureshg@purdue.edu

Cooling Technologies Research Center,

NSF IUCRC, School of Mechanical Engineering and Birck Nanotechnology Center,

Purdue University,

West Lafayette, IN 47907-2088

\begin{abstract}
Optimization under uncertainty (OUU) is a powerful methodology used in design and optimization to produce robust, reliable designs. Such an optimization methodology, employed when the input quantities of interest are uncertain, yields output uncertainties that help the designer choose appropriate values for input parameters to produce safe designs. Apart from providing basic statistical information, such as mean and standard deviation in the output quantities, uncertainty-based optimization produces auxiliary information, such as local and global sensitivities. The designer may thus decide the input parameter(s) to which the output quantity of interest is most sensitive, and thereby design better experiments based on just the most sensitive input parameter(s). Another critical output of such a methodology is the solution to the inverse problem, i.e., finding the allowable uncertainty (range) in the input parameter(s), given an acceptable uncertainty (range) in the output quantities of interest. We apply optimization under uncertainty to the problem of heat transfer in fin heat sinks with uncertainties in geometry and operating conditions. The analysis methodology is implemented using DAKOTA, an open-source design and analysis kit. A response surface is first generated which captures the dependence of the quantity of interest on inputs. This response surface is then used to perform both deterministic and probabilistic optimization of the heat sink, and the results of the two approaches are compared. [DOI: 10.1115/1.4007669]
\end{abstract}

Keywords: uncertainty quantification, optimization under uncertainty, heat transfer, electronics cooling, DAKOTA, generalized polynomial chaos, sensitivity analysis, design of experiments

\section{Introduction}

Uncertainties in engineering design are common and unavoidable. These uncertainties may result from an inherent variability in inputs (aleatory/irreducible uncertainty), such as uncertain geometric parameters and operating conditions, or from a lack of knowledge (epistemic/reducible uncertainty), such as those due to unknown physical phenomena [1]. The challenge then is to efficiently quantify these uncertainties and include them in optimization procedures to produce designs meeting the prescribed reliability and robustness levels.

A conventional (deterministic) optimization approach to an engineering problem in the presence of such uncertainties is to treat the uncertain variables as certain, i.e., assuming them to be fixed at their means or bounds (if the extent/nature of uncertainty is known) and proceeding with the optimization. Once the optimal design parameters are arrived at, a factor of safety may be then included, based on prior experience, to take the inherent variability and other uncertainties into consideration. This approach is simple, easy to implement and computationally inexpensive, and remains the most widely used method. However, in the presence of large uncertainties, or when there are strict constraints (such as expensive design parts, or designs where overall mass is critical), the method fails to produce a truly optimal design that is robust and reliable in addition to being optimal. The conventional approach does not quantify uncertainties and fold them into the optimization procedure; it accounts for them heuristically through a factor of safety.

To perform OUU, optimization techniques must be combined with uncertainty quantification (UQ) procedures. The OUU approach may be used for both "design for robustness" and/or "design for reliability" [2]. The goal of the former design problem

\footnotetext{
${ }^{1}$ Corresponding author.

Manuscript received April 1, 2012; final manuscript received June 20, 2012; published online December 6, 2012. Assoc. Editor: Akshai Runchal.
}

is to produce a design that is less sensitive to variable inputs, such as a design with a low standard deviation in the output quantities. In contrast, in the latter problem, the objective is to produce designs with a lower probability of failure; for this problem, performance statistics at the tails of the distributions are of interest. An example of the latter problem is arriving at designs such that the probability of the output parameter falling below a threshold value is reduced. The former design problem is often the simpler one to address of the two and does not always require UQ [2]. Robust design approaches neglecting UQ rely on local derivatives to assess robustness and are hence not recommended. In the latter problem, UQ is performed and design parameters are estimated based on the tail (end) statistics of the output probability density function (PDF). This problem places a greater demand on UQ and is often computationally more expensive compared to the former problem.

Optimization of electronics cooling equipment has been widely studied [3]. With the advent of high heat-dissipating and densely packed electronics, there is increasing need to optimize the existing cooling solutions and to design newer and more efficient ones. To date, air cooling remains the most widely used approach, owing to its inherent simplicity, low cost, and low maintenance [4]. Extended surfaces, such as pin-fins and parallel-plate fins, aid the heat transfer from a hot surface to the surroundings and are often employed in conjunction with air cooling [5]. Optimizing dimensional parameters, such as fin-to-fin spacing and number of fins for a given set of input parameters is a commonly studied optimization problem in heat transfer. Bar-Cohen and Rohsenow [6] and Bejan [7] developed correlations for optimal spacing between parallel plates for natural convection heat transfer. Similar studies for forced convection were performed by Nakayama et al. [8] and Bejan and Sciubba [9]. Ledezma et al. [10] demonstrated the existence of an optimal spacing between pin fins in crossflow and impinging flow. Based on theoretical arguments relating to similarity of flow and heat transfer, the form of the correlation was borrowed from previous studies on cross-flow over 


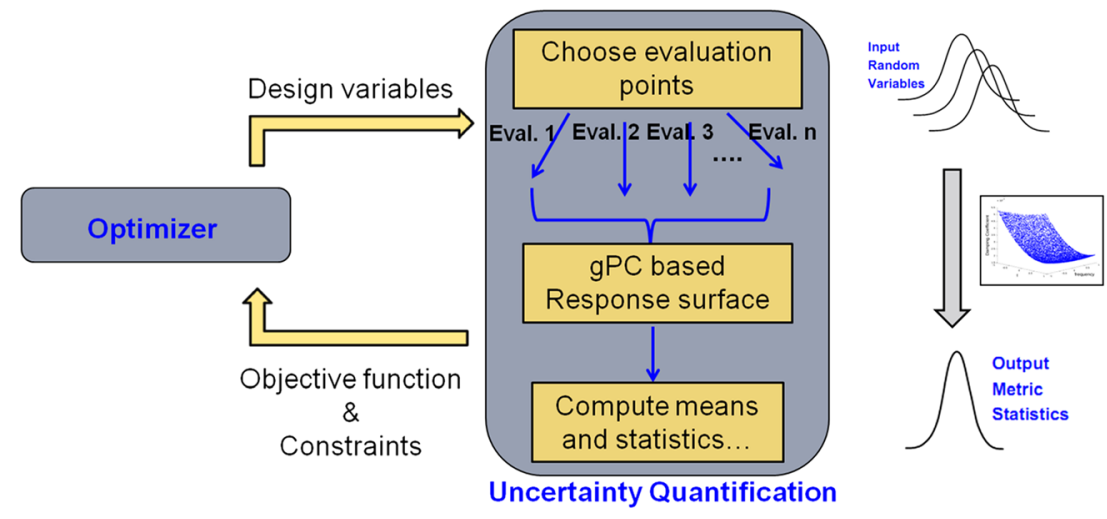

Fig. 1 Nested approach to optimization under uncertainty employed in this work

staggered cylinders [7] and flow between parallel plates [9]. Some of the recent studies on optimization of pin-fin heat sinks include those by Kang [11] and Khan et al. [12].

These optimization studies neglect the uncertainties in the problem being investigated, such as uncertain fin dimensions and the variability in applied heat flux. The objective of the present study is to assess the importance of considering these uncertainties. Optimization under uncertainty is performed for the representative case of impinging flow on pin fins considered by Ledezma et al. [10]. Problem parameters, such as base and fin dimensions and flow speeds, are matched with Ref. [10]. The OUU framework developed here is verified against simple analytical and 2D problems, such as flow in a channel with uncertain viscosity $[13,14]$. The deterministic computational model is first verified for the flow speeds and dimensions considered by Ledezma et al. [10]. The pin-fin geometry is then optimized by considering uncertainties in the geometric and operating conditions. The cost of multiple flow and heat transfer simulations is alleviated by using response functions derived from generalized polynomial chaos (gPC) [15]. Optimization under uncertainty is performed, and robust designs restricting the standard deviation in the Nusselt number are obtained.

\section{Methodology}

In this section, the UQ methodology and the subsequent nested approach to OUU are briefly described. Readers are referred to comprehensive literature on the subject (e.g., Refs. [15] and [1]) for a detailed discussion.

2.1 Uncertainty Quantification. UQ refers to the process of determining the effect of input uncertainties on the output quantities of interest. These uncertainties, as previously mentioned, are generally categorized as either aleatory (i.e., resulting from irreducible, inherent variability) or epistemic (resulting from a lack of knowledge, and thus potentially reducible) [1]. Aleatory uncertainties are typically addressed using probabilistic methods. Approaches to epistemic uncertainty vary. Both probabilistic (Bayesian) methods as well as nonprobabilistic methods have been used to quantify these uncertainties. In the present work, the analysis is restricted to aleatoric uncertainty, to which probabilistic methods, such as polynomial chaos expansions (PCE), may be successfully applied.

Random sampling techniques are the most common UQ methods. Based on the input PDF, samples are drawn randomly based on algorithms, such as Monte Carlo (MC) and Latin hypercube sampling (LHS). For each sample drawn, the function evaluation (simulation/analytical) is performed and when the entire variation of the input variable is covered, the PDF of outputs may be computed along with various other response statistics [1]. Thousands of evaluations must be performed to cover the entire range of the input PDF; the number of evaluations becomes exceptionally large as the number of uncertain variables increases. Other

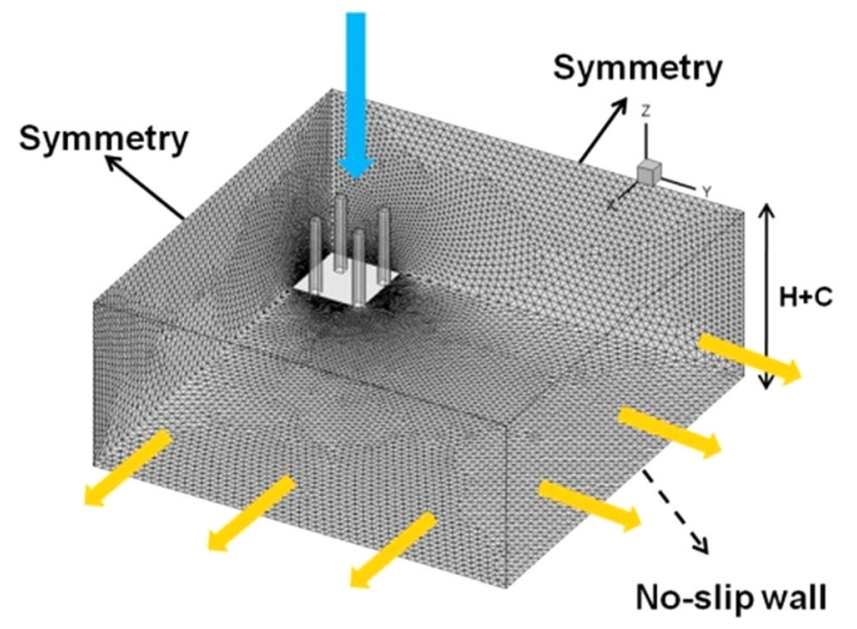

a)

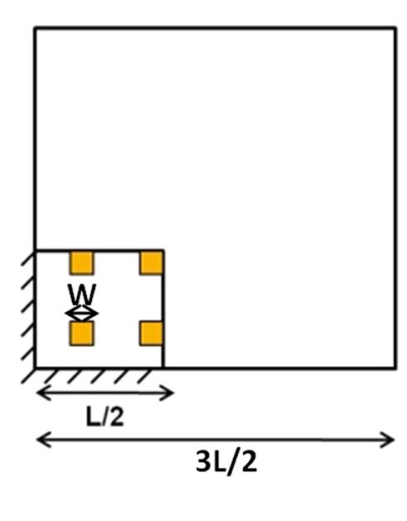

b)

Fig. 2 (a) Isometric view with boundary conditions and (b) top view of pin-fin geometry considered 
methods, such as the sensitivity method based on moments of samples, exist but are less robust and depend on the model assumptions [1].

A more computationally tractable UQ method is the PCE method. In this work, we employ the gPC approach, using the Wiener-Askey scheme originally proposed by Xiu and Karniadakis [15]. In the gPC approach, Hermite, Legendre, Laguerre, Jacobi, and generalized Laguerre orthogonal polynomials are used to model the effect of uncertain variables described by normal, uniform, exponential, beta, and gamma PDFs, respectively, for the input variables. These orthogonal polynomial selections are shown to be optimal for these distribution types since the inner product weighting function and its corresponding support range correspond to the PDF for these continuous distributions [15]. In theory, this selection of the optimal basis allows for exponential convergence rates, which is far better compared to the random sampling techniques mentioned previously [1]. Both intrusive and nonintrusive variants have been proposed [15].

The stochastic collocation method is a nonintrusive method based on gPC [15]. In this approach, the set of polynomials previously mentioned are used as an orthogonal basis to approximate the functional form between the stochastic response output and each of its random inputs. Deterministic simulations are performed at the collocation points in random space and exploitation of the orthogonality properties of the polynomial basis functions allows the determination of the coefficients in the polynomial expansion. Details may be found in Ref. [15] and in the comprehensive review by Eldred [1].

The chaos expansion for a response $R$ takes the form

$$
R=\sum_{j=0}^{\infty} \alpha_{j} \psi_{j}(\xi)
$$

Each of the terms $\psi_{j}(\xi)$ consists of multivariate polynomials which involve products of the corresponding one-dimensional polynomials in the random variable, $\xi$. In practice, the number of terms in Eq. (1) is truncated, and hence only a finite number of evaluations are needed to calculate the polynomial representation of the response function $R$. A number of options exist for choosing the specific evaluation points $j$, such as quadrature grids for each random variable (which translates to a tensor product grid in multiple dimensions, i.e., when there is more than one random variable) and sparse gridding techniques [1]. One such sparse grid technique called the Smolyak sparse grid has proven to be highly efficient, requiring fewer computations than a tensor product grid when there is a large number of uncertain parameters. We employ a Smolyak sparse grid in this work.

Once the response function is constructed based on deterministic runs at the collocation points determined by the Smolyak algorithm, it is then used as a surrogate model. PDFs of the response $R$ may be computed by sampling the space of input random variables using random sampling algorithms, such as MC and LHS. The output response statistics, for example, the mean, standard

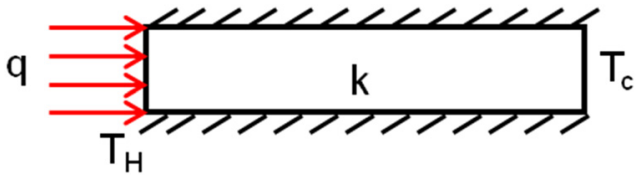

Fig. 3 Schematic diagram of the heater block design problem

deviation, or indeed, the entire output PDF, may then be evaluated. Some of the other auxiliary information that may be obtained from such UQ analysis is the sensitivity information. Based on the reported Sobol' and Kucherenko's indices [16], information such as the sensitivity of a particular output metric to a particular uncertain input parameter may be obtained, which aids in the design of future experiments (DOE). For example, based on this type of UQ analysis and the sensitivity information thus obtained, the not-so-sensitive parameters may be considered as deterministic (certain). Subsequent (refined) UQ may then be performed by resolving the more sensitive parameters more accurately, thereby reducing the computational effort significantly. In this work, we use the open-source UQ and optimization toolkit, DAKOTA [17], for performing the uncertainty quantification and the subsequent optimization.

2.2 Optimization Under Uncertainty. OUU refers to the optimization of a design by taking into consideration the uncertain input parameters and the corresponding output response statistics. Eldred et al. [2] provided an overview of the various OUU formulations available in DAKOTA. Of these, we use the most direct approach called the nested approach to OUU in the present work. The UQ loop is nested within the optimization loop. Starting with initial guess values for the design variables (i.e., initial conditions for the optimization loop), a complete uncertainty quantification is performed using the gPC UQ methodology described in Sec. 2.1. Based on the output response metrics from the UQ loop, the optimizer evaluates the objective function and the corresponding constraints (if it is a constrained optimization problem, such as a design to restrict the standard deviation in the outputs) and checks for convergence. If the convergence criterion is not met, new values for the design variables are selected and the optimization proceeds. The nested OUU approach used in this work is shown schematically in Fig. 1.

At convergence, the set of design variables satisfying a given set of constraints and also simultaneously minimizing (or maximizing) an objective function of interest is obtained. DAKOTA is equipped with a number of gradient and nongradient-based optimization algorithms. We choose the gradient-based optimization algorithm known as the Fletcher-Reeves conjugate gradient method for unconstrained optimization and the method of feasible directions for constrained optimization, based on the CONMIN library [18]. Also, it may be noted that the method is not just limited to global optimization; DAKOTA is equipped with optimizers to deal with multiple, local optima, in addition to the global ones.

Table 1 Optimization results for heater block design problem

\begin{tabular}{lccc}
\hline \hline Parameter & $\begin{array}{c}\text { Analytical solution } \\
\text { (Eq. (6)) }\end{array}$ & $\begin{array}{c}\text { Deterministic } \\
\text { optimization }\end{array}$ & Probabilistic optimization \\
\hline$q$ & 17.0 & 17.0 & Mean = 17.0 and standard deviation $=7.0$ \\
$k$ & 100.0 & 100.0 & Mean $=100.0$ and standard deviation $=30.0$ \\
$T_{\mathrm{c}}$ & 325.0 & 325.0 & Mean $=325.0$ and standard deviation $=25.0$ \\
Initial value of $A\left(\mathrm{~mm}^{2}\right)$ & - & 20.0 & 20.0 \\
Initial value of $L\left(\mathrm{~mm}^{2}\right)$ & - & 10.0 & 10.0 \\
Final value of $A\left(\mathrm{~mm}^{2}\right)$ & 42.50 & 42.48 & 47.44 \\
Final value of $L\left(\mathrm{~mm}^{3}\right.$ & 5.0 & 5.0 & 5.0 \\
Volume $(A \times L)\left(\mathrm{mm}^{3}\right)$ & 212.5 & 212.4 & 237.2 \\
\hline \hline
\end{tabular}




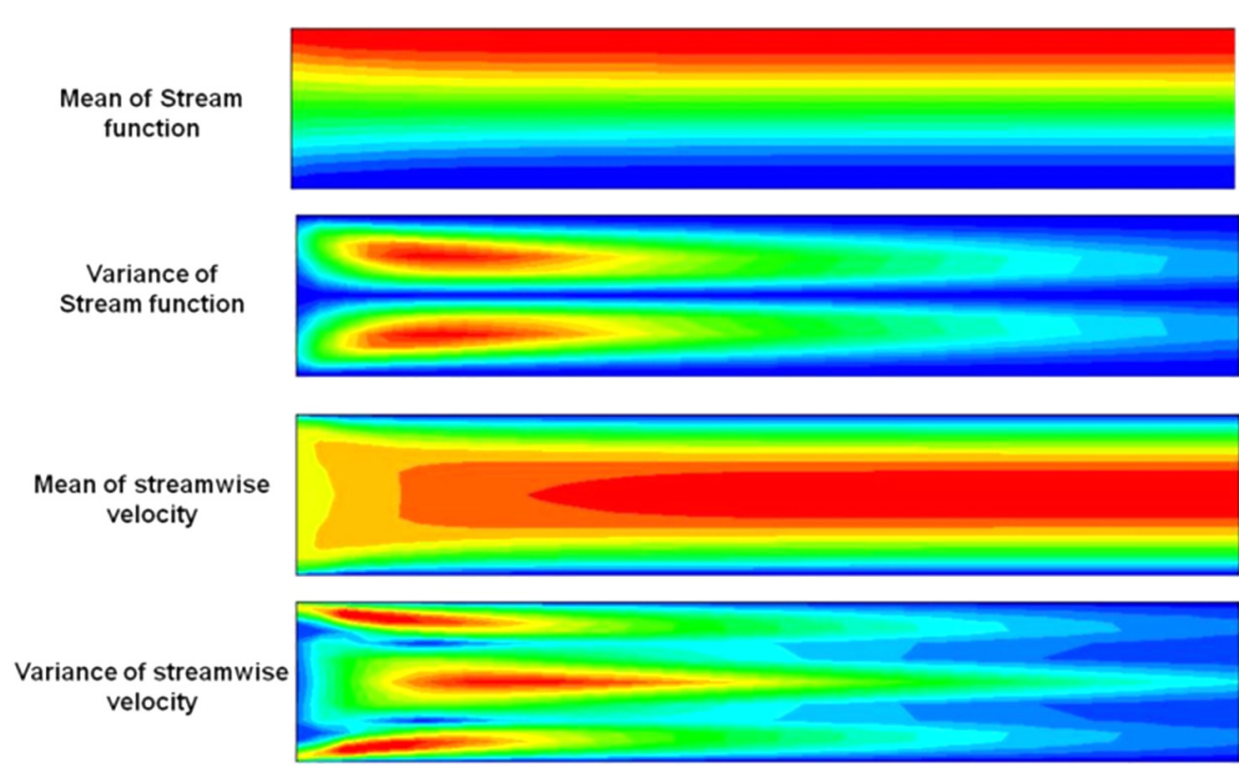

a)


b)

Fig. 4 Two-dimensional channel flow with uncertain viscosity for the case of $R e=81.24$ : (a) current results and $(b)$ results from Le Maitre et al. [14]

\section{Numerical Modeling}

This section describes the governing equations and boundary conditions and details of the numerical model.

3.1 Governing Equations and Boundary Conditions. The continuity, momentum, and energy equations for the steady, constant-property flow of an incompressible Newtonian fluid are given by

$$
\begin{gathered}
\frac{\partial}{\partial x_{i}}\left(\rho u_{i}\right)=0 \\
\frac{\partial}{\partial x_{j}}\left(\rho u_{j} u_{i}\right)=-\frac{\partial P}{\partial x_{i}}+\mu\left(\frac{\partial^{2} u_{i}}{\partial x_{j} x_{j}}\right)
\end{gathered}
$$

$$
\frac{\partial}{\partial x_{i}}\left(\rho C u_{i} T\right)=\frac{\partial}{\partial x_{i}}\left(k \frac{\partial T}{\partial x_{i}}\right)
$$

The computational domain is shown in Fig. 2. Exploiting symmetry, only a $1 / 4$ th section need be considered. The flow enters the computational domain at the top, impinges on the fin surfaces, cools the fins, and leaves the domain at the two outlets along the sides on which the pressure is prescribed. The computational domain is suitably extended in the wake of the pin fins to account for flow reversal. Following Ledezma et al. [10], the fins are assumed to be isothermal, and hence only the fluid domain is considered for analysis. Based on their prior study [19] and other studies on air-cooled heat sinks used in the industry (for example, 


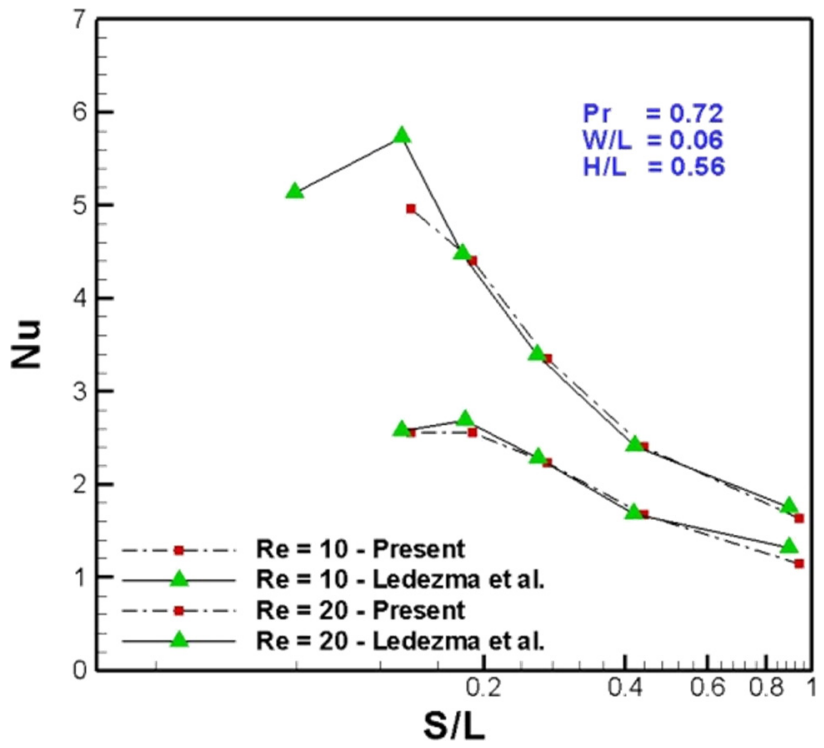

Fig. 5 Nusselt number versus nondimensional fin spacing. The results from Ledezma et al. [10] are shown for comparison.

see Refs. [8] and [20]), Ledezma et al. [10] assert that for an optimization analysis, the isothermal assumption yields results similar to those with a nonisothermal fins assumption. This is an approximation for modeling convenience, and for a more thorough analysis, the solid domain must also be considered in the framework developed as part of the present work. A no-slip boundary condition is prescribed for all the solid-fluid interfaces. Figure 2 shows the various boundary conditions employed in the pin-fin heat transfer problem considered in this study.

3.2 Solution Procedure. For performing the optimization under uncertainty effectively, the computational sequence shown in Fig. 1 must be automated. We use the in-built preprocessor in DAKOTA called DPREPRO [17] for the purpose. Once the computational model (meshing and subsequent analysis) is parameterized, actual values for the parameters may be obtained directly using DPREPRO, with little or no human intervention. The whole process is automated using a simple script written in Python. Also, for efficient computations, the inner-loop UQ evaluations at the Smolyak collocation points are conducted in parallel, thereby speeding up the overall procedure. Furthermore, for the deterministic simulations at the Smolyak collocation points, we use the parallelized computational fluid dynamics (CFD) capabilities of FLUENT [21]. User-defined functions are written for generating the outputs in the format required by DAKOTA. After the first outerloop iteration (which requires many inner-loop UQ evaluations), the results are passed back to the optimizer, which then decides the next set of design variables. The process is repeated until the convergence criterion and the constraints are satisfied.
The geometry is parameterized for meshing, and the journaling features of the meshing package CUBIT [22] employed for generating the meshes at the Smolyak collocation points. The actual values for the parameters in the parameterized journal file are updated using DPREPRO for individual function evaluations. The governing equations are solved using the commercial CFD package FLUENT [21] as described earlier. The equations are first solved with a first-order upwind scheme for a few iterations. Using the flow and temperature fields so obtained as initial conditions, the equations are solved with a second-order upwind scheme until convergence. Pressure-velocity coupling is addressed via the SIMPLE algorithm, along with an algebraic multigrid algorithm for solving the linearized system of governing equations. The governing equations are suitably under-relaxed to ensure proper convergence. Also, the default convergence criteria in FLUENT, based on the scaled residuals, are reduced by two orders for all the governing equations to enforce a stricter convergence criterion. Further details of the numerical method may be found in Ref. [23].

A mesh-independence study is performed by considering three levels of mesh refinement and analyzing the percentage error in the overall Nusselt number with respect to the finest mesh size. This study is conducted at the nominal design point. The number of elements for the three levels for the case of $5 \times 5$ fins is approximately $238,000,514,000$, and $1,455,000$, respectively. It is observed that the relative percentage difference in Nusselt number between the coarse mesh and the finest mesh is approximately $0.75 \%$, while that between the intermediate and the finest mesh is $0.44 \%$. The results from the intermediate mesh are assumed sufficiently accurate for the purposes of this paper, and unless otherwise stated, the results reported in this work are based on this mesh.

A critical parameter of interest in optimization studies is the computational time. With parallelized CFD solvers and mesh sizes of about 0.5 million, each inner-loop evaluation required approximately $1.5 \mathrm{~h}$ of real time employing 3 Intel E5410 CPUs. The computational time also depends on the level of Smolyak grid, which determines the number of inner-loop evaluations per outer-loop evaluation. With 32 available CPUs, we could perform 10 simulations in parallel at any time, leading to a total time of approximately $10-12 \mathrm{~h}$ per optimization run, corresponding to $70-80$ total evaluations (inner loop $\times$ outer loop). A good way to reduce the computational time is to first optimize using the deterministic approach, locate the optimum input parameters, and then begin the probabilistic optimization with these optimal inputs as the initial guessed values. Further reduction in computational time may be realized by assessing the required Smolyak grid level a priori, such as by just performing the uncertainty quantification analysis, and measuring the mean and standard deviation values of the outputs, predicted employing various sparse grid levels. A little change in the response function values with increase in sparse grid level would then mean that lower sparse grid levels would suffice. Following this, in the present work, a sparse grid level of 1 was found to be sufficient, as will be discussed.

Table 2 Uncertainty quantification for pin-fin heat sinks

\begin{tabular}{lcc}
\hline \hline Parameter & Deterministic approach & Probabilistic approach \\
\hline Inputs & & \\
$L(\mathrm{~mm})$ & 45.0 & Uniform random, minimum $=40.0$ and maximum $=50.0$ \\
$t(\mathrm{~mm})$ & 3.5 & Uniform random, minimum $=2.0$ and maximum $=5.0$ \\
$W(\mathrm{~mm})$ & 3.5 & Uniform random, minimum $=2.0$ and maximum $=5.0$ \\
$H(\mathrm{~mm})$ & 20.0 & Uniform random, minimum $=10.0$ and maximum $=30.0$ \\
$c(\mathrm{~mm})$ & 10.0 & Uniform random, minimum $=8.0$ and maximum $=12.0$ \\
$n$ & 5 & 5 \\
Outputs & & Mean $=16.124$ and standard deviation $=6.99$ \\
$\mathrm{Nu}$ & 15.70 & Mean $=2.04$ and standard deviation $=0.92$ \\
$\Delta P(\mathrm{~Pa})$ & 1.79 & \\
\hline \hline
\end{tabular}




\section{Results and Discussion}

We now present results for the uncertainty quantification and optimization under uncertainty for three test cases.

4.1 Heater Block Design. The first problem considered in this study corresponds to a simple heater block design, for which an analytical solution exists. This example problem was considered as a verification case for the OUU framework.

The basic setup of the problem is shown in Fig. 3. The uncertain parameters considered in this problem are the heat input $q$, the thermal conductivity of the heater block $k$, and the temperature at the cold end $T_{\mathrm{c}}$. The block is assumed to be insulated at the sides and the objective is to design the block with minimum volume, $A \times L$. Here, $A$ is the area of cross section and $L$ is the length. Additional constraints in the problem are posed by restricting the mean value of the temperature at the hot end to a design temperature value of $345 \mathrm{~K}$. The uncertain variables are considered to be Gaussian random variables, i.e., normally distributed with mean and standard deviation values as shown in Table 1 . The constrained minimization problem may be formally stated as follows:

$$
\begin{aligned}
& \text { Minimize } A \times L \\
& \text { suchthat } \overline{T_{\mathrm{h}}} \leq T_{\text {design }} \\
& 10.0 \leq A \leq 100.0 \\
& 5.0 \leq L \leq 20.0
\end{aligned}
$$

For fixed values of the uncertain variables, the temperature value at the hot end, $T_{\mathrm{h}}$, may be calculated as follows [5]:

$$
T_{\mathrm{h}}=T_{\mathrm{c}}+\frac{q L}{k A}
$$

4.1.1 Deterministic Optimization. As a first step, the optimization is performed for the case of deterministic values for the uncertain variables. For this, the uncertain parameters, $q, k$, and $T_{\mathrm{c}}$, are all fixed at their mean values. The initial values for the optimization loop are chosen to be 10 and 20 for $L$ and $A$, respectively. The final optimized values for the design variables $A$ and $L$ are shown in Table 1. It may be noted that the values correspond very well with the analytical solution, obtained by setting $T_{\mathrm{h}}=T_{\text {design }}$ and choosing $A$ by fixing $L$ or vice versa. Also, the optimization process is assumed converged if the value of the objective function changes by less than $0.1 \%$ for three or more successive iterations.

4.1.2 Probabilistic Optimization. For probabilistic optimization, uncertainties are considered as part of the optimization procedure, as explained earlier. Beginning with the same initial guess values as for the case of the deterministic optimization, function evaluations for the inner UQ loop are performed. A Smolyak sparse grid of level 2 as described in Ref. [17] is used for this purpose and based on evaluations at the collocation points, the polynomial response surface is obtained. This response surface is then used as a surrogate model and samples based on the LHS algorithm are drawn to cover the input variable range. The corresponding output values are estimated for each drawn sample and once a sufficient number of samples are drawn, output response metrics, such as the mean output value may be obtained. The mean $T_{\mathrm{h}}$ value thus calculated is used by the optimizer to evaluate the inequality constraint in Eq. (5). The process is repeated until convergence; the convergence criterion used here is the same as in the case of the deterministic optimization. The corresponding optimized values for the design variables are shown in Table 1. It may be observed that, for the probabilistic case, design variables assume more conservative values. In particular, for the present problem, an approximately $12 \%$ larger volume is recommended from the probabilistic case as compared to the deterministic one. The OUU approach thus allows the user to obtain a quantitative estimate of how conservative the design should be to account for uncertainties in the problem and is more robust compared to a heuristic factor-of-safety approach.

4.2 Channel Flow With Uncertain Viscosity. A 2D channel flow with uncertain viscosity was considered by Le Maitre et al. [14]. This was one of the first studies which investigated the use of polynomial chaos UQ methods for incompressible laminar flow at moderate Reynolds numbers, as noted by Najm [13]. Le Maitre et al. [14] used an intrusive PCE method to modify the governing equations, Eqs. (2)-(4) to account for uncertain viscosity. Also, they employed a finite difference-based projection method for solving the governing equations. In the present work, we use the same problem (problem P1 from Ref. [14]) to verify our modeling framework. The problem statement is briefly summarized here and additional details may be found in Ref. [14]. We note that the stochastic projection method of Le Maitre et al. is an intrusive PCE method, for which the governing equations must be modified as must the corresponding solver for performing the UQ [13]. However, we use a nonintrusive PCE method and use the original

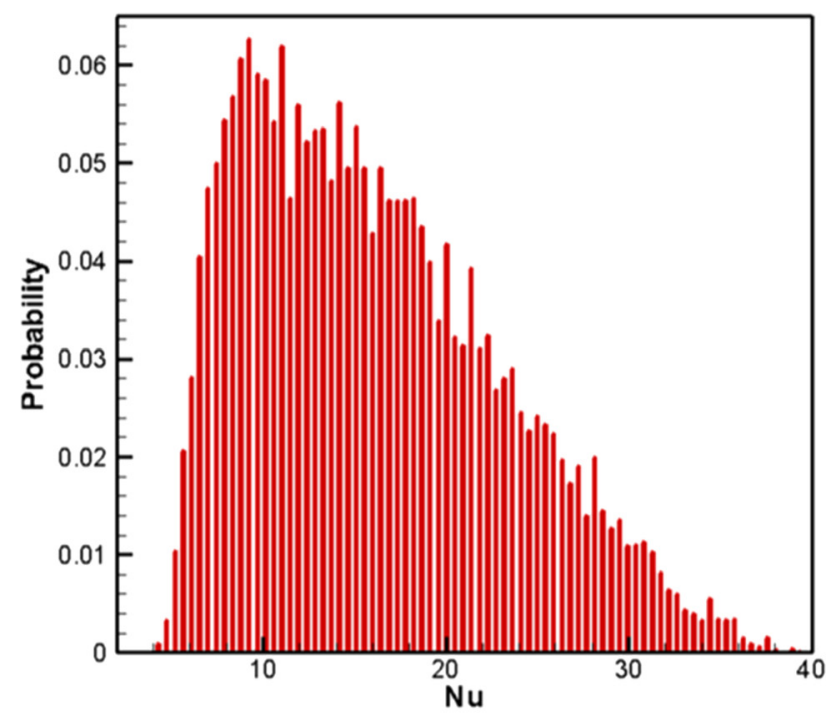

a)

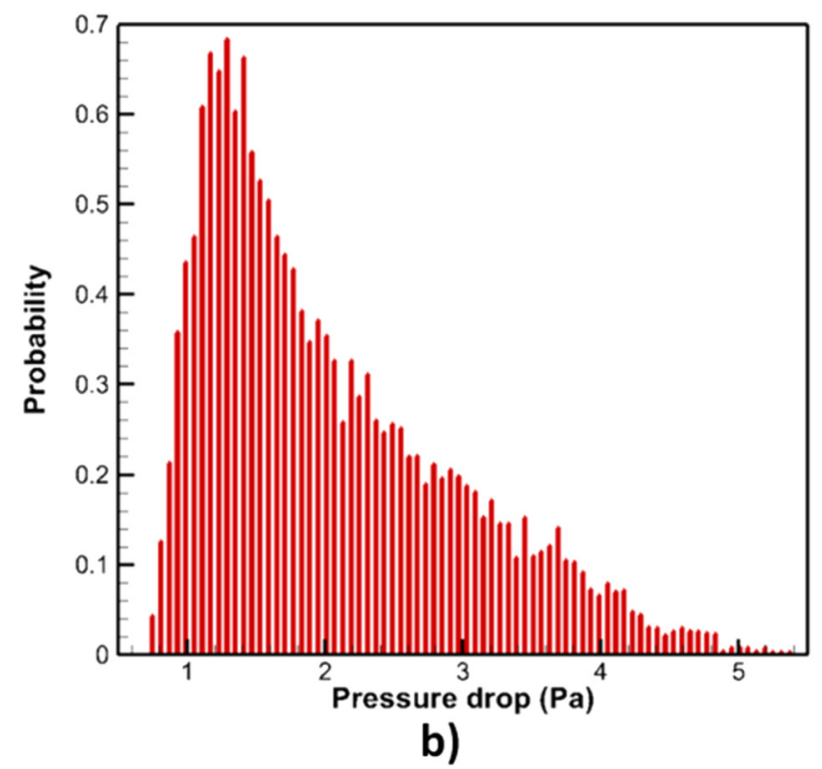

Fig. 6 PDFs of (a) Nusselt number and (b) pressure drop for the uniformly distributed input parameters in Table 2 


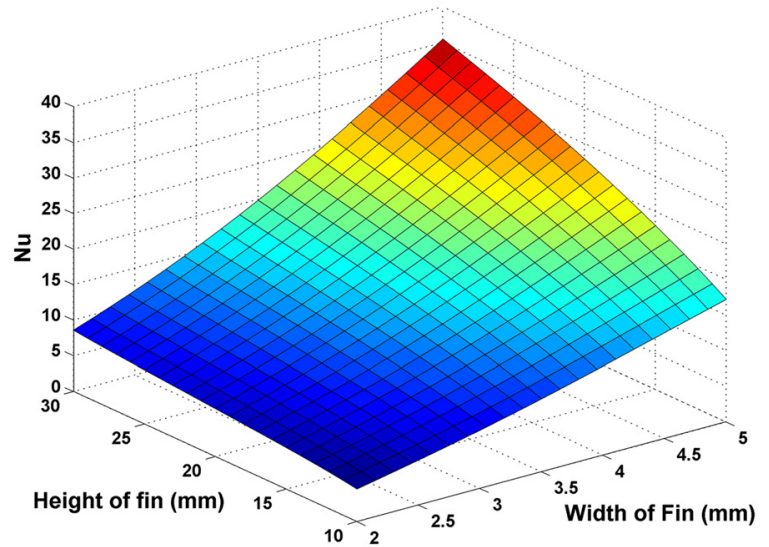

a)

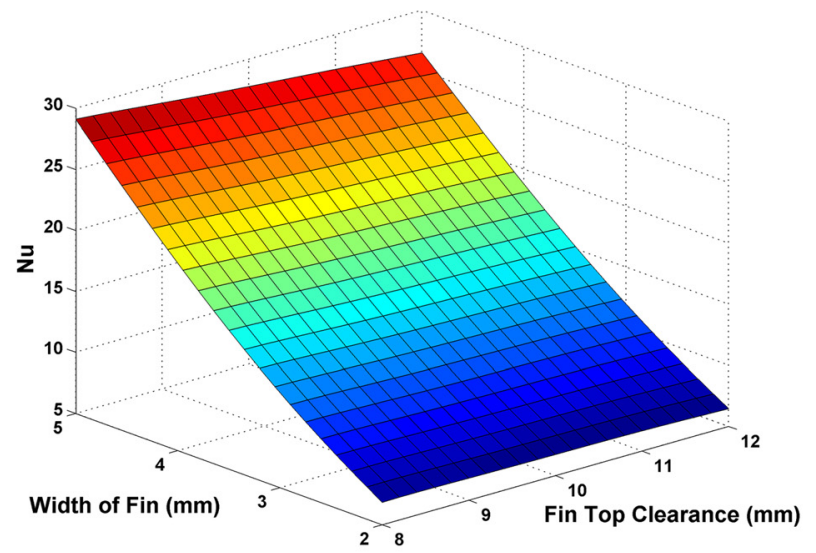

b)

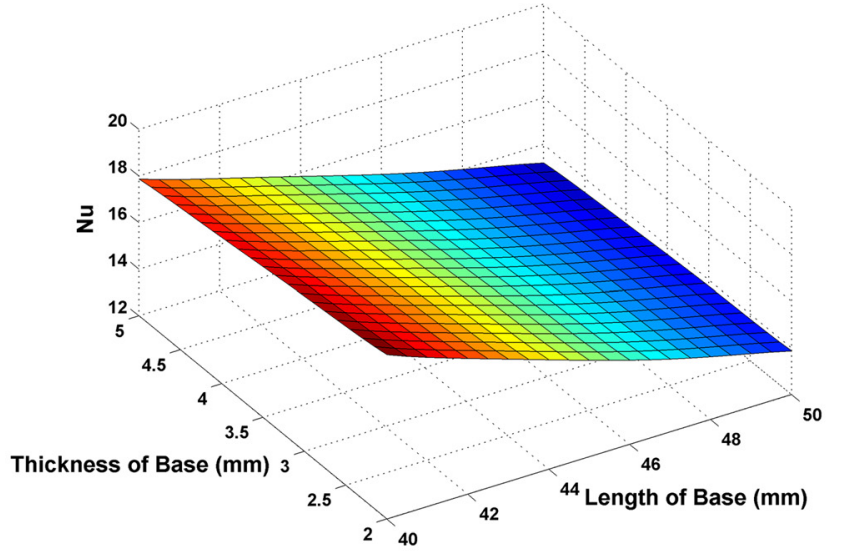

c)

Fig. 7 Response surface plots of Nusselt number as a function of various input parameters

governing equations (Eqs. (2)-(4)) and the standard commercial solver, FLUENT.

The viscosity is assumed to be spatially uniform. At each location in the domain, it is considered to be a Gaussian random variable with a predefined mean and standard deviation. The boundary conditions however are deterministic, i.e., a constant, uniform flow boundary condition is imposed at the inlet, while a no-slip condition is imposed on the two bounding walls. The flow is developing initially but attains fully developed flow conditions some distance downstream of the inlet. Figure 4 shows the contours of the mean and variance of stream function and streamwise velocity for a Reynolds number of 81.24 and a ratio of standard deviation to mean of the kinematic viscosity of 0.2 , as used in Ref. [14]. It may be observed that the results match qualitatively with those of Le Maitre et al. and this loosely verifies our framework. The development length, as inferred from the velocity data, is found to be about four times the channel width, which is in close agreement with theory $\left(\mathrm{x}_{\mathrm{fd}} / \mathrm{l} \approx 0.05 \mathrm{Re},[5]\right)$ and the results of Le Maitre et al., which further verifies our results.

In the preceding two examples, we have demonstrated that UQ can be performed nonintrusively and in a computationally inexpensive manner. The approach adopted here requires fewer function evaluations than other UQ methods. The process is nonintrusive in that new stochastic solvers, such as those used by Le Maitre et al. [14] are not needed. Instead, uncertainties are quantified through the output-input response function approach described.

4.3 Pin-Fin Optimization. The final problem we consider in this work pertains to the optimization of the width of the pin-fins cooled by impinging flow in the presence of uncertainties.
At a constant input velocity of impingement, a optimum pin spacing exists [10]. As the spacing between the fins tends to zero (infinite fin limit), there is little penetration of flow between the fins, and the fins are underutilized. Similarly, for a very large fin spacing (zero fin limit), the reduction in surface area leads to a reduced amount of heat transfer. This leads to the existence of an optimum spacing (see, for example, Refs. [9] and [19]). Ledezma et al. [10] performed a numerical optimization of the fin spacing for an impinging flow configuration. Their results were first validated against their experiments. The number of fins was then optimized as the design parameter, for different input parameters, such as fin height, width, and Reynolds number. The optimal pin spacing was found to decrease as the Reynolds number increased, and the peak of the Nusselt number versus fin spacing curve flattened as the Reynolds number was decreased. For the range of Reynolds numbers considered, a fin count value of 4 or 5 along the side of the heat sink base was found to be optimal. However, their methodology did not employ a formal optimization procedure. Also, a discrete parameter, the number of fins, was chosen as the design variable, and simulations with different values of the fin count were conducted to arrive at an optimum.

In the present work, the number of fins is fixed at $n=5 \times 5$ fins spanning the entire base area for the optimization problem and the continuous parameter, the fin width $W$, is optimized using a more formal optimization approach based on the Fletcher-Reeves conjugate gradient method [17]. Simulations are first performed for the set of conditions considered by Ledezma et al. [10], viz., a nondimensional fin width $W / L$ of 0.06 , a nondimensional fin height $H / L$ of 0.56 , and a Prandtl number of 0.72 . The simulations are performed for two different Reynolds numbers and the results, along with those from Ledezma et al., are shown in Fig. 5. Length 

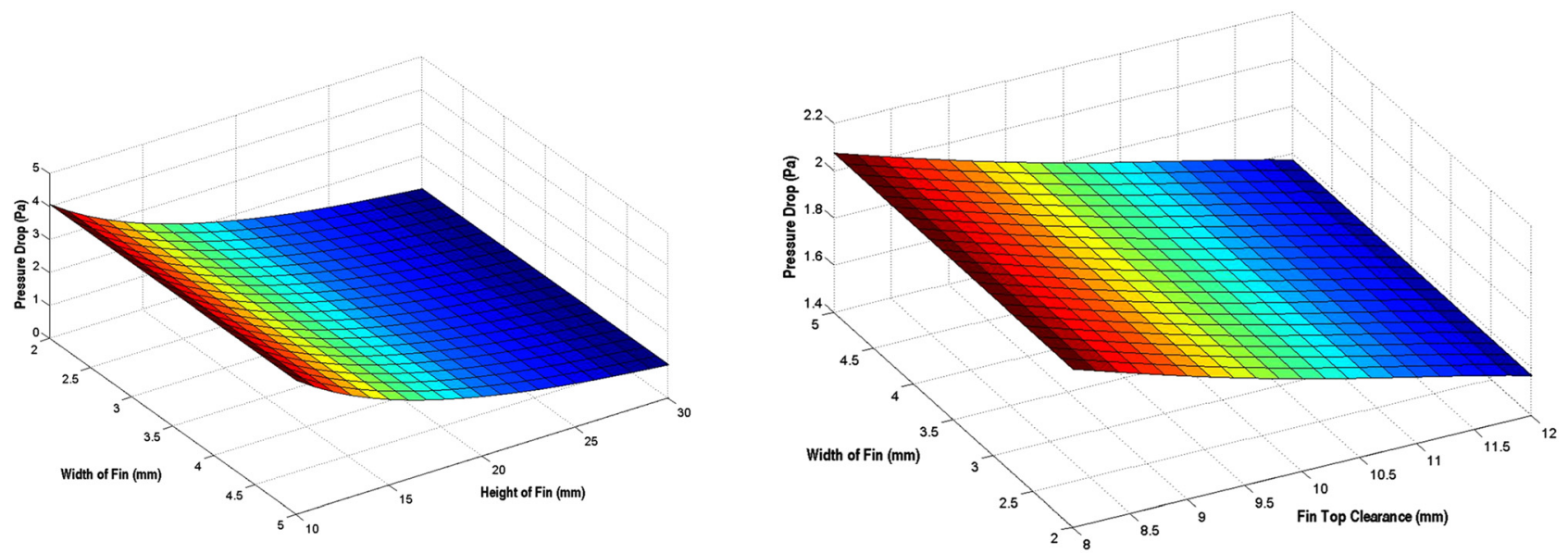

a)

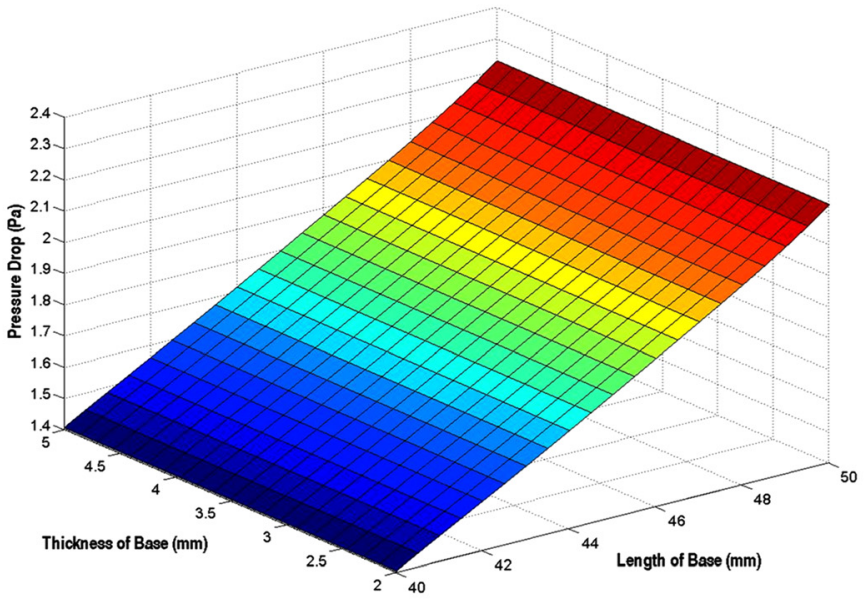

b)

\section{c)}

Fig. 8 Response surface plots of pressure drop as a function of various input parameters

parameters are nondimensionalized by the length of the heat sink base, $L$, consistent with Ledezma et al. So also, the Reynolds number and the Nusselt number are defined based on the fin width, $W$, as follows:

$$
\operatorname{Re}=\frac{\rho U_{i n} W}{\mu} ; \mathrm{Nu}=\frac{q W}{L^{2}\left(T_{\mathrm{s}}-T_{\infty}\right) \times k_{f}}
$$

In Eq. (7), $U_{\mathrm{in}}, T_{\mathrm{s}}$ and $T_{\infty}$ are the inlet velocity, specified fin surface temperature and ambient air stream temperature, respectively.

The small differences between the present results and those from Ledezma et al. as observed in Fig. 5 may be attributed to the coarser meshes considered by Ledezma et al., as well as the uniform, structured nature of the meshes, with very few cells in between the fins. In the present work, we have employed a boundary-layer mesh for resolving the fin surfaces and ensured that there are at least $5-10$ computational cells between successive fins in the cross-flow direction.

4.3.1 Uncertainty Quantification. The input parameters considered for UQ are as follows: length of the base, $L$; thickness of the base, $t$; width of the fin, $W$; height of the fin, $H$; and clearance between the top of the fin and the inlet plane, $c$. These variables are all assumed to be uniformly distributed random variables. The corresponding variation in the input parameters is shown in Table 2. Again the number of fins is fixed at $n=5 \times 5$.

A level 2 Smolyak sparse grid is used for uncertainty quantification which, for 5 uniformly distributed uncertain variables, translates to 71 evaluations for constructing the response surface. The inlet velocity for these runs was fixed at a value of $0.5 \mathrm{~m} / \mathrm{s}$. Following the procedure previously described, once the response surface is obtained, 10,000 samples are drawn randomly and output response statistics are calculated. The PDFs of Nusselt number and pressure drop are plotted in Fig. 6, corresponding to the range

Table 3 Sensitivity analysis for pin-fin heat sinks

\begin{tabular}{lcc}
\hline \hline Variable input parameter & Range of variation in the input parameter & Standard deviation in Nu \\
\hline$L$ & Uniform random, minimum $=40.0$ and maximum $=50.0$ & 1.41 \\
$t$ & Uniform random, minimum $=2.0$ and maximum $=5.0$ & 0.21 \\
$c$ & Uniform random, minimum $=8.0$ and maximum $=12.0$ & 0.35 \\
$H$ & Uniform random, minimum $=2.0$ and maximum $=5.0$ & 6.13 \\
$H$ & Uniform random, minimum $=10.0$ and maximum $=30.0$ & 2.69 \\
\hline \hline
\end{tabular}




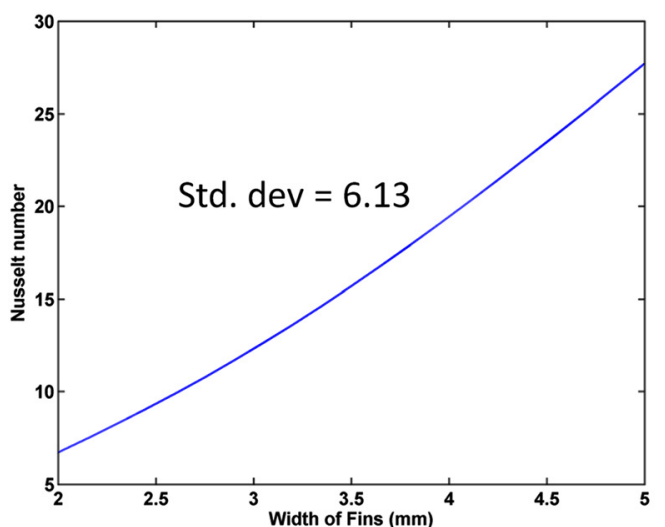

a)

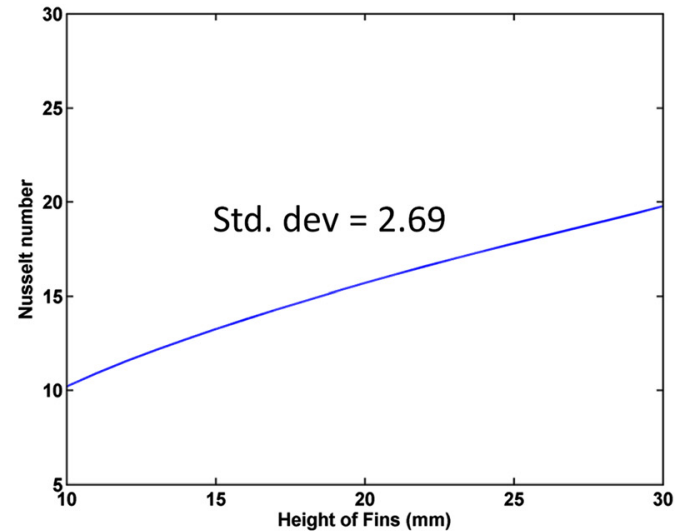

b)

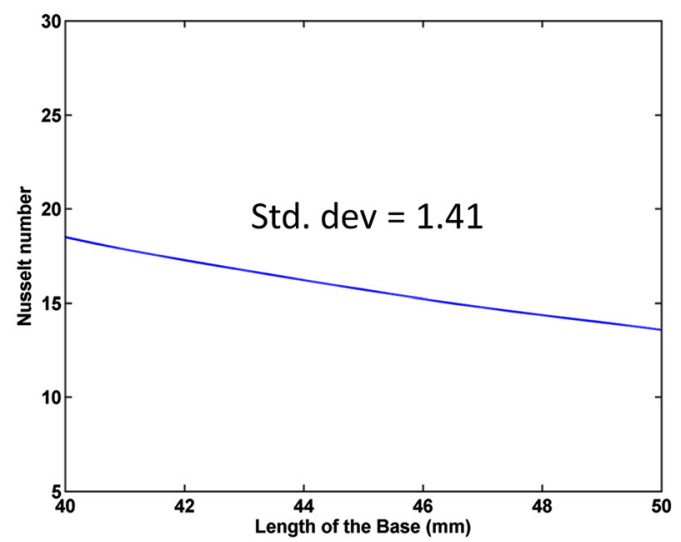

c)

Fig. 9 Plots of Nusselt number shown for three most sensitive input parameters: (a) fin width, (b) fin height, and (c) length of the base, for OUU of pin-fin heat sinks

of the uncertain inputs considered. Representative response surface plots for the outputs, Nusselt number and pressure drop, are shown in Figs. 7 and 8, respectively, as a function of the various input variables. Also, the corresponding mean and standard deviation in the output quantities are reported in Table 2. As a comparison, simulations are also performed by fixing the uncertain variables at their means and the corresponding results are included in Table 2. It may be observed that the mean values of the output variables, as obtained from the UQ simulations, are different from the values obtained by assuming the uncertain variables to be deterministic and assigning them their respective mean values. Also, in order to assess the sensitivity of outputs to the various uncertain inputs, simulations are performed by fixing all input variables at their respective mean values except the input parameter relative to which the sensitivity is being assessed. By doing so, the input variables relative to which the outputs show the highest standard deviation are identified as the most sensitive variables.

Table 3 lists the standard deviation in $\mathrm{Nu}$ as the various input parameters, viz., $L, t, c, W$, and $H$ are varied. Variations in the input variables similar to those in Table 2 are considered. Of all the variables, it is clear that the Nusselt number is most sensitive to variations in the width $W$ and height $H$ of the fins. Sensitivity information such as this is useful in helping the designer decide which input parameters are most important. Such information also aids in the DOE, as noted by Eldred et al. [2]. For further assessing the dependence of outputs on these sensitive input variables, the response surfaces are plotted in Fig. 9. The shape of the response functions from Fig. 9, suggests that a lower-order Smolyak grid is sufficient for this problem, and hence a Smolyak sparse grid of level 1 is employed in this work for the remainder of the results. However, simulations were also performed with a level 2 sparse grid to verify this choice, and similar results, with less than $0.1 \%$ difference, were obtained. The corresponding PDFs of the Nusselt number for the case of single parameter variation are shown in Fig. 10.

We may thus perform an initial approximate UQ analysis using, for example, low-accuracy polynomial expansions, and based on the sensitivity information, the most sensitive parameters may be identified. The uncertainty in only the most sensitive parameters may then be resolved better.

The UQ loop is then nested within an outer optimization loop for probabilistic optimization. To demonstrate the idea, we use an OUU approach for "design for robustness." That is, the optimization is performed to choose the design variables that not only maximize (minimize) the cost function but also restrict the standard deviation in the output quantities, which is posed as an additional constraint in the optimization procedure. Two sets of simulations are performed. In the first case, uncertainties in dimensional parameters are considered, which may arise due to manufacturing limitations. An example for such a case is microchannels, where large deviations in the channel dimensions are observed [24]. In the second case, uncertainties in thermal and flow conditions are considered.

4.3.2 OUU With Uncertain Dimensions. As a first demonstration case, uncertainties in the thickness of the base $t$ and the fintop clearance $c$ are considered for different inlet velocities. The length of the base $L$ and height $H$ are fixed at $47.6 \mathrm{~mm}$ and $26.66 \mathrm{~mm}$, respectively, to match the values from Ref. [10]. Again, uniform uncertain variables, with minimum and maximum values as reported in Table 4, are considered for this analysis. For these sets of simulations, a sparse grid of level 1 is considered for the UQ and optimization is performed, starting with an initial guess value for the width, $W$. 


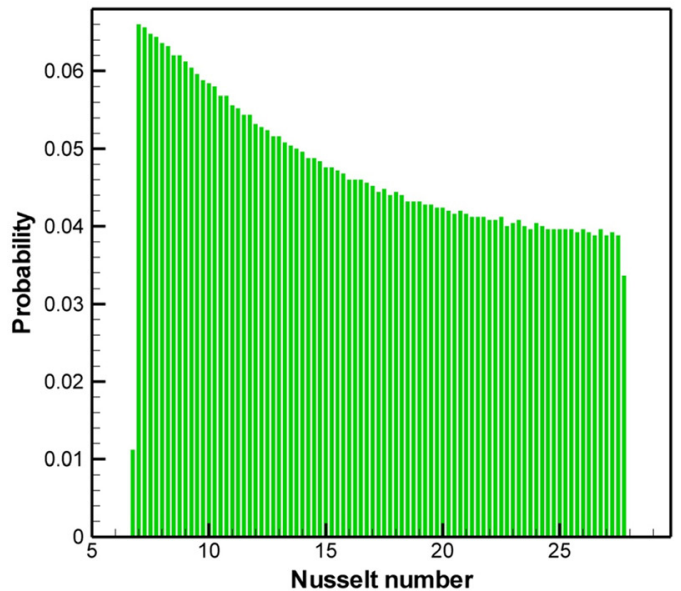

a)



b)

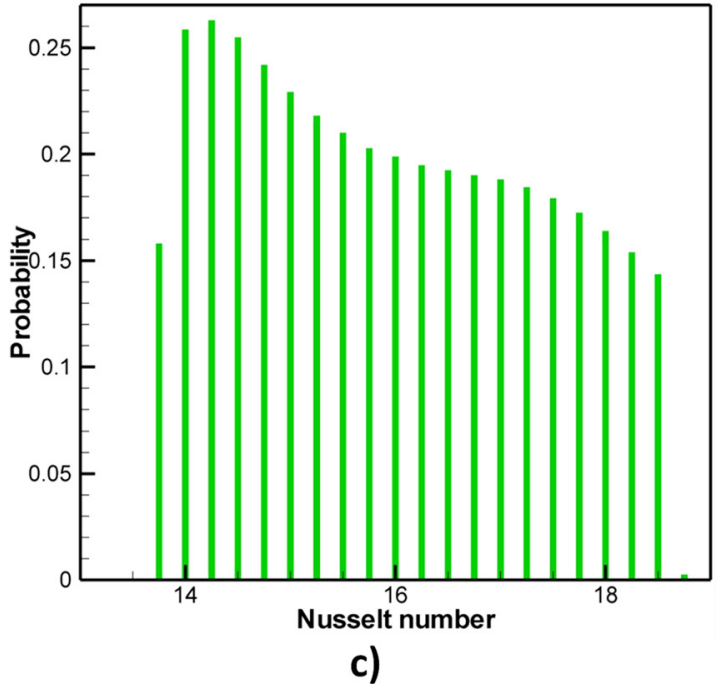

Fig. 10 Nusselt number PDFs shown for the three most sensitive input parameters: (a) fin width, (b) fin height, and (c) length of the base, for OUU of pin-fin heat sinks

For comparison, conventional (deterministic) optimization is also performed for fixed values of the uncertain variables, i.e., with uncertain variables assuming their respective mean values. It may be observed from Table 5 that for the deterministic runs, as the velocity is increased, the optimum width of the fins shifts to higher values, consistent with the observation of Ledezma et al. [10]. This is because at lower velocities, the flow does not penetrate the pins adequately and the increase in fin width is detrimental to the overall heat transfer. On the contrary, at higher velocities, flow penetration is significant even at the larger fin widths and an increase in fin width enhances overall heat transfer by effectively increasing the surface area. For the probabilistic runs, a constraint bounding the standard deviation of the Nusselt

Table 4 Input parameters for optimization in the case of uncertain dimensions

\begin{tabular}{|c|c|c|}
\hline Parameter & $\begin{array}{l}\text { Deterministic } \\
\text { optimization }\end{array}$ & $\begin{array}{l}\text { Probabilistic } \\
\text { optimization }\end{array}$ \\
\hline$L(\mathrm{~mm})$ & 47.6 & 47.6 \\
\hline$t(\mathrm{~mm})$ & 3.5 & $\begin{array}{l}\text { Uniformly distributed with } \\
\text { minimum }=2.0 \text { and maximum }=5.0\end{array}$ \\
\hline$H(\mathrm{~mm})$ & 26.66 & 26.66 \\
\hline$c(\mathrm{~mm})$ & 10.0 & $\begin{array}{c}\text { Uniformly distributed with } \\
\text { minimum }=8.0 \text { and maximum }=12.0\end{array}$ \\
\hline$n$ & 5 & 5 \\
\hline$T_{\mathrm{s}}-T_{\infty}(\mathrm{K})$ & 20.0 & 20.0 \\
\hline
\end{tabular}

number is also applied, and the optimization problem may be defined as follows:

$$
\begin{aligned}
& \text { Maximize } \overline{\mathrm{Nu}} \\
& \text { such that Std.Dev. }(\mathrm{Nu}) \leq 0.5 \\
& 0.48 \mathrm{~mm} \leq W \leq 8.75 \mathrm{~mm}
\end{aligned}
$$

The limits on the design variable $W$ are chosen so as to cover the entire range from very thin fins (infinite spacing limit) to very thick fins (zero spacing limit), with $8.75 \mathrm{~mm}$ being just under the theoretical maximum width (theoretical maximum $=L$ / $5=9.52 \mathrm{~mm}$ ).

The convergence history of the optimization procedure is plotted in Fig. 11 along with results for the deterministic runs. The corresponding converged values for the width are shown in Table 5. It may be noted that the values of Nusselt number shown

Table 5 Optimized width and corresponding Nusselt number in the case of uncertain dimensions. The presented values of Nusselt number for the probabilistic case are mean values and the bold values correspond to the inputs.

\begin{tabular}{lcccccc}
\hline & \multicolumn{3}{c}{ Deterministic optimization } & \multicolumn{3}{c}{ Probabilistic optimization } \\
\hline $\mathbf{U}_{\text {in }}(\mathbf{m} / \mathbf{s})$ & $\mathbf{0 . 0 5}$ & $\mathbf{0 . 2}$ & $\mathbf{0 . 4}$ & $\mathbf{0 . 0 5}$ & $\mathbf{0 . 2}$ & $\mathbf{0 . 4}$ \\
$W(\mathrm{~mm})$ & 6.26 & 7.20 & 7.75 & 6.21 & 5.12 & 4.51 \\
$\mathrm{Nu}$ & 8.19 & 27.91 & 51.84 & 8.23 & 18.34 & 22.57 \\
\hline \hline
\end{tabular}




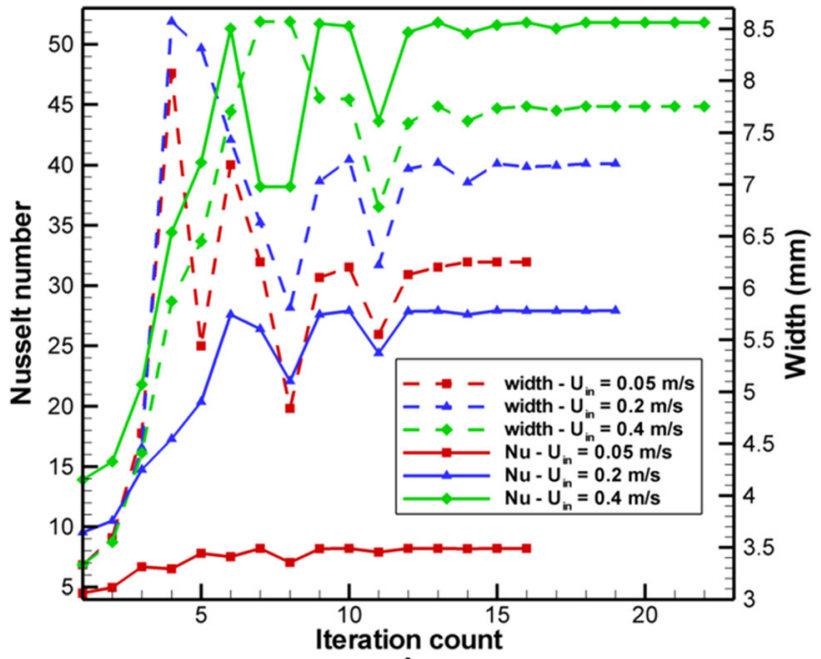

a)

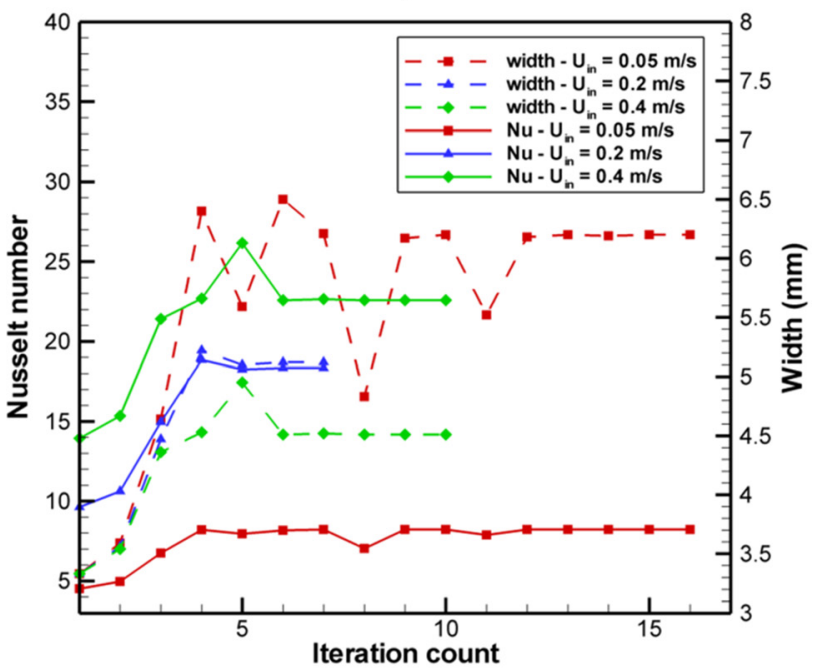

b)

Fig. 11 Convergence history for OUU with uncertain dimensions using (a) deterministic optimization for maximizing Nusselt number and $(b)$ probabilistic optimization for maximizing mean value of Nusselt number

for the probabilistic case are mean values. By restricting the standard deviation in the output parameter, more conservative values result for the design variables. More importantly, the extent to which a design is conservative to account for uncertainties is quantified with this approach. Also, there is a significant reduction

Table 6 Input parameters for optimization for the case of uncertain operating conditions

\begin{tabular}{lcc}
\hline \hline Parameter & $\begin{array}{c}\text { Deterministic } \\
\text { approach }\end{array}$ & $\begin{array}{c}\text { Probabilistic } \\
\text { approach }\end{array}$ \\
\hline$L(\mathrm{~mm})$ & 47.6 & 47.6 \\
$t(\mathrm{~mm})$ & 3.5 & 3.5 \\
$H(\mathrm{~mm})$ & 26.66 & 26.66 \\
$c(\mathrm{~mm})$ & 10.0 & 10.0 \\
$n$ & 5 & 5 \\
$T_{\mathrm{s}}(\mathrm{K})$ & 320.0 & $\begin{array}{c}\text { Normally distributed, with } \\
\text { mean }=320.0 \text { and standard } \\
\text { deviation }=10.0\end{array}$ \\
Mean Re & & $\begin{array}{c}\text { Normally distributed, with } \\
\text { mean }=50.0,100.0,300.0 \\
\text { Standard deviation in Re }\end{array}$ \\
\hline \hline
\end{tabular}

Journal of Heat Transfer

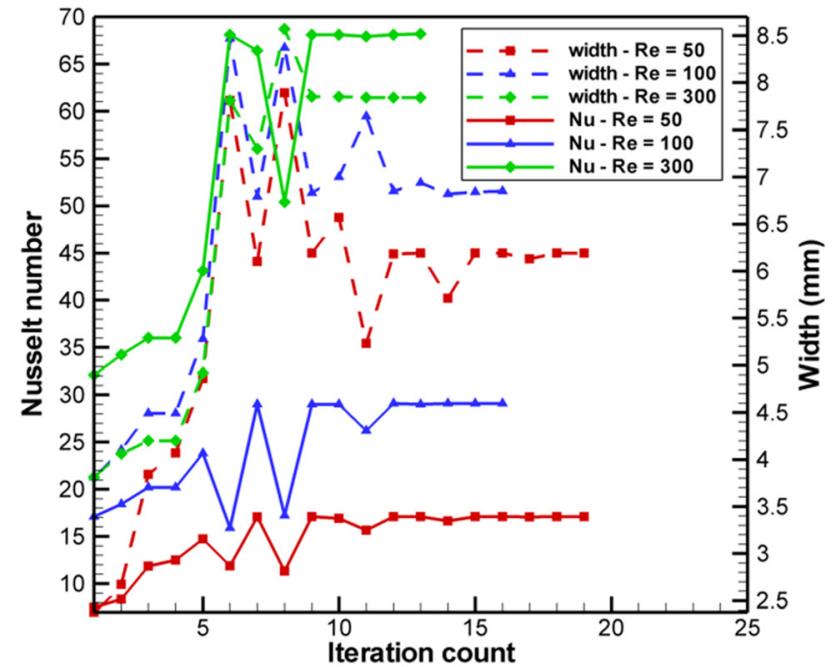

a)

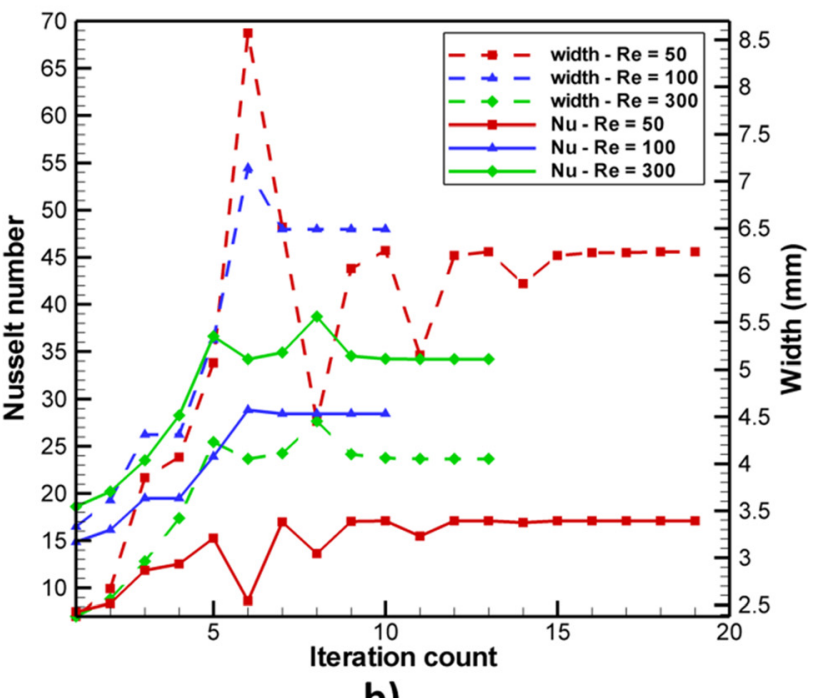

b)

Fig. 12 Convergence history for OUU with uncertain operating conditions using (a) deterministic optimization for maximizing Nusselt number and $(b)$ probabilistic optimization for maximizing mean of Nusselt number

in the overall Nusselt number compared to the deterministic case, which again demonstrates the conservative nature of the design. The information from the convergence history in Fig. 11 may also be used for obtaining a first estimate of the output values for a value of the design variable that is different from the final converged value.

4.3.3 OUU With Uncertain Operating Conditions. As a second demonstration case, uncertainties in the inlet velocity (Reynolds number) and input heat fluxes are considered as in a real application. A desktop computer heat sink, for instance, sees different heat generation levels depending on processor load even if designs are generally based on a thermal design power [25] which is a manufacturer-quoted maximum possible heat dissipation. Similarly, the flow speed from the fan can be uncertain and variable.

As before, we seek to perform uncertainty propagation first and then perform OUU to obtain the optimum fin width. The uncertain parameters are the Reynolds numbers and the pin-fin surface temperature, which are assumed to be Gaussian random variables for this purpose, with mean and standard deviation values as listed in Table 6. As in the previous case of uncertain dimensions, $L$ and $H$ 
Table 7 Optimized width and corresponding Nusselt number for the case of uncertain operating conditions. The presented values of Nusselt number for the probabilistic case are mean values and the bold values correspond to the inputs.

\begin{tabular}{lcccccc}
\hline \hline & \multicolumn{2}{c}{ Deterministic optimization } & \multicolumn{3}{c}{ Probabilistic optimization } \\
\hline $\mathbf{R e}$ & $\mathbf{5 0 . 0}$ & $\mathbf{1 0 0 . 0}$ & $\mathbf{3 0 0 . 0}$ & $\mathbf{5 0 . 0}$ & $\mathbf{1 0 0 . 0}$ & $\mathbf{3 0 0 . 0}$ \\
$W(\mathrm{~mm})$ & 6.19 & 6.85 & 7.84 & 6.25 & 6.49 & 4.05 \\
$\mathrm{Nu}$ & 17.10 & 29.07 & 68.16 & 17.09 & 28.45 & 34.18 \\
\hline \hline
\end{tabular}

values are fixed at $47.6 \mathrm{~mm}$ and $26.66 \mathrm{~mm}$, respectively. In addition, $t$ and $c$ are also held fixed at $2.0 \mathrm{~mm}$ and $10.0 \mathrm{~mm}$, respectively. The standard deviation in the Reynolds number values considered is loosely based on the uncertainty analysis of Ledezma et al. [10], who noted a 5\% uncertainty in Reynolds number in their experiments. A 3\% standard deviation to mean ratio in the solid-fluid interface (fin and base) temperature is considered.

An additional constraint is imposed to restrict the standard deviation of the Nusselt number, as in the previous case. The corresponding optimization problem may be defined as below

$$
\begin{aligned}
& \text { Maximize } \overline{\mathrm{Nu}} \\
& \text { such that Std.Dev. }(\mathrm{Nu}) \leq 1.0 \\
& 0.48 \mathrm{~mm} \leq W \leq 8.75 \mathrm{~mm}
\end{aligned}
$$

The converged results for mean Nusselt number, along with Nusselt number values from the deterministic equivalent, obtained by assuming the mean values for the interface temperature and Reynolds number are shown in Fig. 12 and Table 7. Again, the conservative nature of the probabilistic optimization is clearly observed. For the deterministic case, the value of the optimal fin width is observed to increase with Re as for the OUU with uncertain dimensions. Physical reasons for this behavior were previously explained. However, for the case of probabilistic optimization, due to the additional constraint restricting the standard deviation in $\mathrm{Nu}$, no such clear trends are observed. From Table 7, it may be noted that as long as the constraint is not limiting, such as for $\operatorname{Re}=50$ and $\mathrm{Re}=100$, similar results are obtained with both deterministic and probabilistic approaches. At $\operatorname{Re}=300$, however, a very different optimum value for the width is returned compared to the deterministic case, as otherwise, the constraint would not be satisfied.

\section{Conclusions}

A framework for performing optimization in the presence of uncertain inputs is developed using the UQ and optimization toolkit, DAKOTA. The OUU framework developed here is verified against a test case for which an exact solution exists. The framework is also tested by performing uncertainty quantification for a simple 2D channel flow with uncertain but spatially uniform viscosity. The results are verified against the results from Le Maitre et al. [14].

Optimization of fin spacing in heat sinks is of broad interest in electronics cooling. Traditionally, the input variables are assumed to be deterministic for such an optimization, while in reality there could be uncertainties in both dimensions and operating conditions. In this work, a pin-fin heat sink under an impinging flow, similar to that considered by Ledezma et al. [10], is considered for optimization. Two sets of uncertainties commonly encountered in engineering design problems, viz., uncertainties in dimensions and uncertainties in operating conditions, are explored. The corresponding results are compared with their deterministic counterparts and the conservative nature of the probabilistic designs is quantified. Based on sensitivity information, the critical input parameters to which the output quantities are most sensitive are also identified.

\section{Acknowledgment}

The authors acknowledge the support for this work from industry members of the Cooling Technologies Research Center, an NSF Industry/University Cooperative Research Center.

$$
\begin{aligned}
& \text { Nomenclature } \\
& A=\text { area, } \mathrm{mm}^{2} \\
& c=\text { fin-top clearance, } \mathrm{mm} \\
& C=\text { specific heat, } \mathrm{J} / \mathrm{kg} \mathrm{K} \\
& H=\text { fin height, mm } \\
& k=\text { thermal conductivity, } \mathrm{W} / \mathrm{mK} \\
& L=\text { length of the heat sink base, } \mathrm{mm} \\
& n=\text { number of fins on a side } \\
& \mathrm{Nu}=\text { Nusselt number } \\
& \Delta P=\text { pressure drop, } \mathrm{Pa} \\
& q=\text { heat input, } \mathrm{W} \\
& R=\text { response function } \\
& \mathrm{Re}=\text { Reynolds number } \\
& s=\text { fin-to-fin spacing, mm } \\
& t=\text { thickness of the heat sink base, } \mathrm{mm} \\
& T=\text { temperature, } \mathrm{K} \\
& u=\text { velocity, } \mathrm{m} / \mathrm{s} \\
& W=\text { width of the fin, mm }
\end{aligned}
$$

\section{Greek Symbols}

$\alpha_{i}=$ coefficients in the response function

$\xi_{i}=$ random variable

$\mu=$ fluid dynamic viscosity, $\mathrm{N} \mathrm{s} / \mathrm{m}^{2}$

$\rho=$ density, $\mathrm{kg} / \mathrm{m}^{3}$

$\psi_{i}=$ polynomials in the gPC response function

$$
\begin{aligned}
& \text { Subscripts } \\
& \mathrm{f}=\text { fluid } \\
& \mathrm{h}, \mathrm{c}=\text { hot, cold ends } \\
& \text { in }=\text { inlet } \\
& \mathrm{s}=\text { solid } \\
& \infty=\text { ambient }
\end{aligned}
$$

\section{References}

[1] Eldred, M. S., 2009, "Recent Advances in Non-Intrusive Polynomial Chaos and Stochastic Collocation Methods for Uncertainty Analysis and Design," 50th AIAA/ASME/ASCE/AHS/ASC Structures, Structural Dynamics and Materials Conference, Palm Springs, CA, May 4-7, American Institute of Aeronautics and Astronautics Inc.

[2] Eldred, M. S., Giunta, A. A., Wojtkiewicz, S. F., Jr., and Trucano, T. G., 2002, "Formulations for Surrogate-Based Optimization Under Uncertainty," Proceedings of the 9th AIAA/ISSMO Symposium on Multidisciplinary Analysis and Optimization, Buckhead, Atlanta, GA, Sept. 4-6.

[3] Ndao, S., Peles, Y., and Jensen, M. K., 2009, "Multi-Objective Thermal Design Optimization and Comparative Analysis of Electronics Cooling Technologies," Int. J. Heat Mass Transfer, 52(19-20), pp. 4317-4326.

[4] Kitajo, S., Takeda, Y., Kurokawa, Y., Ohta, T., and Mizunashi, H., 1992 "Development of a High Performance Air Cooled Heat Sink for Multi-Chip Modules," 8th Annual IEEE Semiconductor Thermal Measurement and Management Symposium, New York, NY, Feb. 3-5, pp. 119-124.

[5] Incropera, F. P., and De Witt, D. P., 2002, Fundamentals of Heat and Mass Transfer, John Wiley \& Sons, New Jersey.

[6] Bar-Cohen, A., and Rohsenow, W. M., 1984, "Thermally Optimum Spacing of Vertical, Natural Convection Cooled, Parallel Plates," ASME J. Heat Transfer, 106(1), pp. 116-123.

[7] Bejan, A., 2004, Convection Heat Transfer, John Wiley \& Sons, New York.

[8] Nakayama, W., Matsushima, H., and Goel, P., 1988, "Forced Convective Heat Transfer From Arrays of Finned Packages," Cooling Technology for Electronic Equipment, Hemisphere, Washington, DC, pp. 195-210.

[9] Bejan, A., and Sciubba, E., 1992, "The Optimal Spacing of Parallel Plates Cooled by Forced Convection," Int. J. Heat Mass Transfer, 35(12), pp. 3259-3264. 
[10] Ledezma, G., Morega, A. M., and Bejan, A., 1996, "Optimal Spacing Between Pin Fins With Impinging Flow," ASME J. Heat Transfer, 118, pp. 570-577.

[11] Kang, H. S., 2010, "Optimization of a Pin Fin With Variable Base Thickness," ASME J. Heat Transfer, 132(3), p. 034501.

[12] Khan, W. A., Culham, J. R., and Yovanovich, M. M., 2005, "Optimization of Pin-Fin Heat Sinks Using Entropy Generation Minimization,” IEEE Trans. Compon. Packag. Technol., 28(2), pp. 247-254.

[13] Najm, H. N., 2009, "Uncertainty Quantification and Polynomial Chaos Techniques in Computational Fluid Dynamics," Annu. Rev. Fluid Mech., 41, pp $35-52$.

[14] Le Maitre, O. P., Knio, O. M., Najm, H. N., and Ghanem, R. G., 2001 “"A Stochastic Projection Method for Fluid Flow," J. Comput. Phys., 173(2), pp. 481-511.

[15] Xiu, D., and Karniadakis, G. E., 2002, "The Wiener-Askey Polynomial Chaos for Stochastic Differential Equations,” SIAM J. Sci. Comput. (USA), 24(2), pp. 619-644.

[16] Sobol', I. M., and Kucherenko, S., 2009, "Derivative Based Global Sensitivity Measures and Their Link With Global Sensitivity Indices," Math. Comput. Simul., 79(10), pp. 3009-3017.

[17] Eldred, M. S., Giunta, A. A., van Bloemen Waanders, B. G., Wojtkiewicz, S. F., Hart, W. E., and Alleva, M. P., 2007, DAKOTA, a Multilevel Parallel Object-Oriented Framework for Design Optimization, Parameter Estimation,
Uncertainty Quantification, and Sensitivity Analysis: Version 4.1 Reference Manual, Sandia National Laboratories, Albuquerque, NM.

[18] Vanderplaats, G. N., 1973, CONMIN, a FORTRAN Program for Constrained Function Minimization: User's Manual, Ames Research Center and US Army Air Mobility R\&D Laboratory, Moffett Field, CA.

[19] Morega, A. M., Bejan, A., and Lee, S. W., 1995, "Free Stream Cooling of a Stack of Parallel Plates,” Int. J. Heat Mass Transfer, 38(3), pp. 519-531.

[20] Matsushima, H., Yanagida, T., and Kondo, Y., 1992, "Algorithm for Predicting the Thermal Resistance of Finned LSI Packages Mounted on a Circuit Board," Heat Transfer-Jpn. Res., 21(5), pp. 504-517.

[21] User's Guide for FLUENT 6.0, 2002, Fluent Inc., Lebanon, NH

[22] CUBIT 10.0 User's Manual, 2005, Sandia National Laboratories, Albuquerque, NM.

[23] Bodla, K. K., Murthy, J. Y., and Garimella, S. V., 2010, "MicrotomographyBased Simulation of Transport Through Open-Cell Metal Foams," Numer. Heat Transfer, Part A, 58(7), pp. 527-544.

[24] Lee, P.-S., Garimella, S. V., and Liu, D., 2005, "Investigation of Heat Transfer in Rectangular Microchannels," Int. J. Heat Mass Transfer, 48(9), pp. 1688-1704.

[25] Ganapathy, D., and Warner, E. J., 2008, "Defining Thermal Design Power Based on Real-World Usage Models," 2008 11th IEEE Intersociety Conference on Thermal and Thermomechanical Phenomena in Electronic Systems (ITHERM), Orlando, FL, May 28-31, IEEE, pp. 1242-1246. 